\title{
The Laser Welding Image Registration Method Based on Quaternion and Geometric Moment
}

\author{
Liu Feipeng ${ }^{1, a^{*}}$, Hua Liang ${ }^{1, b}$, Gu Juping ${ }^{1, c}$, Liu Yuqing ${ }^{1}$, Qiang Yujian ${ }^{1}$ \\ ${ }^{1}$ School of Electrical Engineering, Nantong University, Nantong, Jiangsu, 226019, China \\ ${ }^{a}$ liufp_123@163.com, ${ }^{b}$ hualiang@ntu.edu.cn, cgu.jp@ntu.edu.cn
}

Keywords: Laser weld, Image registration, Quaternion, Geometric moment.

Abstract. Considering the angle deviation of the molten pool images that is caused by the different position of multiple cameras in the process of the laser welding image acquisition, this paper put forward a kind of new image registration method based on quaternion and geometric moment, which expressed the molten pool images in the form of quaternion first, and then calculated the main shaft and the mass of the two images to be registered, getting the angle and displacement difference between the two images, finally realized two welding image registration work. Experiments showed that the method was simple and effective. It could fuse the difference among welding images caused by different imaginative angles.

\section{Introduction}

Nowadays, the latest laser welding technology has many bright spots compared with the traditional welding method [1-2], so it has begun to slowly spread in various fields. However, the welding quality cannot be determined by only detecting the parameters of laser welding machine in the welding process, such as laser power and welding speed. What's more, it is difficult to take full advantage of the high efficiency of laser welding to detect the quality of the work piece after welding. In order to ensure the welding quality, numerous scholars have detected the molten pool and keyhole dynamic behavior [3-5]. They explored the physics mechanism and interaction between them to guide the welding process and improve the quality of welding. In research applications, it is difficult to meet the required data of the researches to consider only one modal image. It is hoped that more adequate data information can be provided by different modals of the images, which is taken as a reference to reduce misjudgment and improve the characteristics accuracy of the same scene. Therefore, developing a registration method, which has the characteristics of high precision, strong robustness and so on, has become the constantly struggle direction of image registration researchers [6-7]. In this paper, a new kind of laser welding image registration method based on quaternion and geometric moment is put forward.

\section{Laser welding image registration}

\section{The theoretical basis}

Taking the R, G, B three components as the three imaginary components of a quaternion respectively, this article treats the whole molten pool image as a physical entity, and the two-dimensional geometric moment of the image can be written as $m_{p q}$ [8]:

$$
m_{p q}=\sum_{M} \sum_{N} x^{p} y^{q} f(x, y),(p, q=0,1,2 \mathrm{~K}), m_{p q}, f(x, y) \in H .
$$

Where, $p+q$ is the time of geometric moment and $x=1,2,3 \ldots M, y=1,2,3 \ldots N$.

The geometric moment expression form of the image expressed with quaternion contains not only the global feature information of the image color and shape, but the geometry characteristics which can distinguish different types of image. When $p+q$ takes different values, $m_{p q}$ can represent different physical significanc: 
1) The zeroth-order moment of the quaternion

When $p=q=0, m_{00}$ is called as the zeroth-order moment of the quaternion and use it to represent the color total of the whole image. If each pixel of a color image has quality, $m_{00}$ will be the quality total of the image in each pixel and the "quality" is a vector.

2) The first-order moment of the quaternion

$m_{01}$ is the first-order color matrix of the image on the $x$ axis and $m_{10}$ on $y$ axis. And $\left(x_{0}, y_{0}\right)$ represents the position of the color image mass in the physical sense. The so-called "mass" $\left(x_{0}, y_{0}\right)$ is as follows:

$$
\left(x_{0}, y_{0}\right)=\left(\frac{\left|m_{10}\right|}{\left|m_{00}\right|}, \frac{\left|m_{01}\right|}{\left|m_{00}\right|}\right) \text {. }
$$

Where $|\bullet|$ is to calculate the quaternion matrix module and also represents the calculation of the color intensity.

3) The central moment of the quaternion

The geometric moment of all pixel points relative to $\left(x_{0}, y_{0}\right)$ in a color image is called the central moment of the image matrix, which is shown below:

$$
\mu_{p q}=\sum_{M} \sum_{N}\left(x-x_{0}\right)^{p}\left(y-y_{0}\right)^{q} f(x, y) .
$$

Where, $\mu_{00}=m_{00}, \mu_{01}=\mu_{10}=0$.

4) The second-order moment of the quaternion

The second-order moment is the geometric moment set $m_{p q}$ when $p=q=2$. At this point, the set $m_{p q}$ represents a measure, which is used to evaluate about the transformation of the image color distribution in the origin. The second-order moments $\mu_{02}$ and $\mu_{20}$ are the variance of "mass" and the covariance is given by $\mu_{11}$. The second-order moment can be used to describe the size and direction of the image.

According to the second-order moments $\{\mu 11, \mu 20, \mu 02\}$ of quaternion, the ellipse-like features of image can be achieved by an ellipse which centers the "mass" and has the same image "quality", image direction and eccentricity of the image and the ellipse has a constant emissivity. The geometric distribution expression of an ellipse-like molten pool image is given in Fig. 1. The welding pool images using in this paper are taken from Reference [9]. As shown in Fig. 1, the shafts $a$ and $b$ are the long axis and short axis of the ellipse, respectively. In this paper, the long axis $a$ is taken as the main shaft of the whole image, and the angle $\alpha$ between the long axis $a$ and positive direction of $x$ axis is defined as the main shaft direction. The ellipse-like image is essentially a color image, and the quaternion ellipse-like representation of the color image is a kind of color information in specific ways. Use $c$ to represent it, then:

$$
c=\frac{\mu_{00}}{\pi a b} .
$$
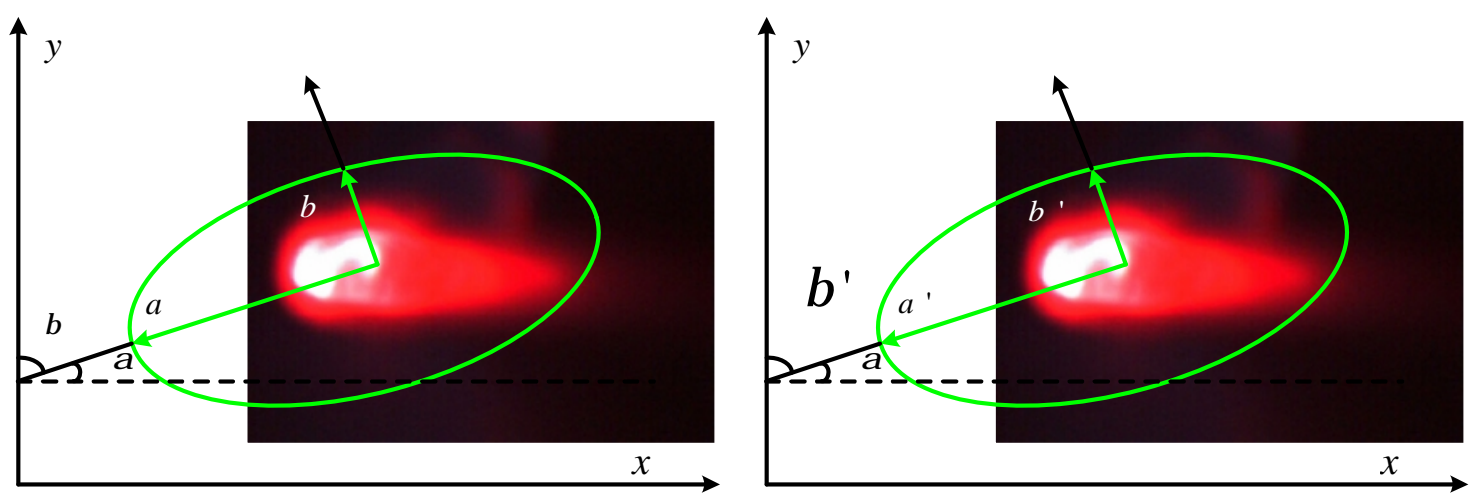

Fig. 1 The molten pool and its ellipse-like representation (left) and that after $3^{\circ}$ rotation (right)

\section{Registration method}

The registration theory used in this paper and the theoretical basis is from Reference [10]. The method in Reference [10] is used for laser welding image registration in this paper, and good results 
have been achieved. In order not to lose vector data information of the color image between R, G, B the three channels in registration, a molten pool registration method is presented by using quaternion to represent the pixel values of molten pool images. This method firstly uses quaternion vector matrix to express the pixels of laser welding images, and then calculates the geometric moments of the quaternion. After that, it uses the geometric algebra algorithm based on quaternion to calculate the masses and the spindles of two images, and then calculates the translation amount $(\Delta x, \Delta y)$ between the images according to the Euclidean distance between the masses and the rotation angle too, according to the spindles. After all these work, the required registration parameters can be got. Thus, the registration work between two different modes of color welding images can be realized.

As shown in Fig. 1, the rotation angle $\varphi$ between two images can be calculated by subtracting $\alpha$ from $\alpha^{\prime}$, that is $\varphi=\left|\alpha-\alpha^{\prime}\right|$. Through the study, it is known that the main shaft direction is closely connected with the rotational inertia of the image. As a result, the concepts of rotational inertia of quaternion image need to be introduced. The expression of rotational inertia is shown as follows:

$$
I=\left[\begin{array}{cc}
I_{x x} & -I_{x y} \\
-I_{y x} & I_{y y}
\end{array}\right] .
$$

Where, $I_{x x}=\left|\sum_{x, y}\left[\left(y-y_{0}\right)^{2}\right] f(x, y)\right|, I_{x y}=\left|\sum_{x, y, z}\left[\left(x-x_{0}\right)\left(y-y_{0}\right)\right] f(x, y)\right|, I_{y y}=\left|\sum_{x, y}\left[\left(x-x_{0}\right)^{2}\right] f(x, y)\right|$.

$I_{x y}=I_{y x}$, so $I$ has the nature of the real symmetric matrix. The rotational inertia is given in the form of quaternion module, containing the color intensity information, which can represent the image characteristics of color distribution.

Thus, the rotational inertia relative to any axis $L$ of the surface of the two-dimensional image which is in form of quaternion can be expressed as:

$$
I_{L}=I_{y y} \cos ^{2} \beta+I_{x x} \cos ^{2} \alpha-2 I_{x y} \cos \alpha \cos \beta \text {. }
$$

Where, $\alpha, \beta$ are respectively the included angles between axis $L$ and the positive direction of $x$ axis and $y$ axis. As they are shown in Fig. 2:

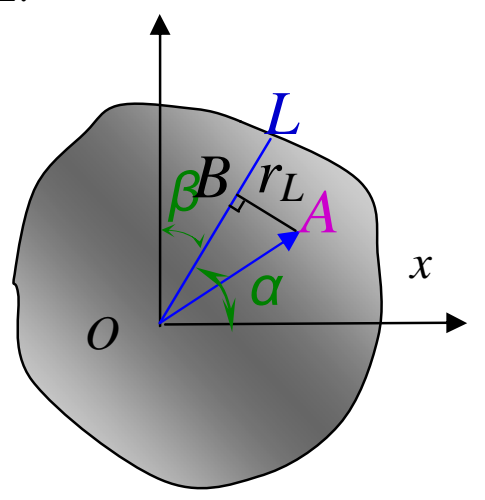

Fig. 2 The schematic diagram of the rotation

Proof: Because the rotational inertia $I_{L}$ of the object relative to $L$ is:

$I_{L}=\sum_{x, y} f(x, y) r_{L}^{2}$.

Where, $r_{L}^{2}=(O A)^{2}-(O B)^{2} . O B$ is the projection of $O A$ in the axis $L$, so

$\pm O B=x \cos \alpha+y \cos \beta$.

And because $(O A)^{2}=x^{2}+y^{2}$, so $r_{L}^{2}=\left(x^{2}+y^{2}\right)-(x \cos \alpha+y \cos \beta)^{2}$.

Because $\cos ^{2} \alpha+\cos ^{2} \beta=1$, then:

$r_{L}^{2}=\left(x^{2}+y^{2}\right)\left(\cos ^{2} \alpha+\cos ^{2} \beta\right)-(x \cos \alpha+y \cos \beta)^{2}$

$r_{L}^{2}=y^{2} \cos ^{2} \alpha+x^{2} \cos ^{2} \beta-2 x y \cos \alpha \cos \beta$

Then the rotational inertia relative to $L$ can be concluded as: 


$$
I_{L}=\sum_{x, y} f(x, y) r_{L}^{2}=\sum_{x, y} f(x, y) y^{2} \cos ^{2} \alpha+\sum_{x, y} f(x, y) x^{2} \cos ^{2} \beta-2 \sum_{x, y} f(x, y) x y \cos \alpha \cos \beta .
$$

According to Ep. 10, the following equation can be got:

$I_{L}=I_{x x} \cos ^{2} \alpha+I_{y y} \cos ^{2} \beta-2 I_{x y} \cos \alpha \cos \beta$.

Because $I_{L}$ has strong links with $a$ axis direction and $b$ axis direction of the ellipse-like image, which has the maximum in the $a$ axis direction and the minimum in the $b$ axis direction, so the corresponding direction angle $\alpha$ of the image main axis can be determined according to Ep. 12:

$$
\alpha=1 / 2 \tan ^{-1}\left(\frac{2\left|\mu_{11}\right|}{\left|\mu_{20}\right|-\left|\mu_{02}\right|}\right) \text {. }
$$

Proof: according to Ep. 5 and $\alpha+\beta=\frac{\pi}{2}$, seeing from Fig. 2, the following equation can be got:

$I_{L}=I_{x x} \cos ^{2} \alpha+I_{y y} \sin ^{2} \alpha-2 I_{x y} \cos \alpha \sin \alpha$.

Where, $I_{x x}=\left|\sum_{x, y}\left[\left(y-y_{c}\right)^{2}\right] f(x, y)\right|=\left|\mu_{02}\right|, I_{x y}=\left|\sum_{x, y}\left[\left(x-x_{c}\right)\left(y-y_{c}\right)\right] f(x, y)\right|=\left|\mu_{11}\right|$. So Ep. 13 can be written as:

$$
I_{L}=\left|\mu_{02}\right| \cos ^{2} \alpha+\left|\mu_{20}\right| \sin ^{2} \alpha-2\left|\mu_{11}\right| \cos \alpha \sin \alpha .
$$

When $I_{L}$ gets to the maximum, according to $\alpha$, the direction of the main shaft can be known. Calculate the derivative of $\alpha$ in Ep. 14:

$$
\begin{aligned}
& \frac{d I_{L}}{d \alpha}=\frac{d\left(\left|\mu_{02}\right| \cos ^{2} \alpha+\left|\mu_{20}\right| \sin ^{2} \alpha-2\left|\mu_{11}\right| \cos \alpha \sin \alpha\right)}{d \alpha}=0 \\
& \Rightarrow\left(\left|\mu_{20}\right|-\left|\mu_{02}\right|\right) \sin 2 \alpha-2\left|\mu_{11}\right| \cos 2 \alpha=0 \\
& \Rightarrow \tan 2 \alpha=\frac{2\left|\mu_{11}\right|}{\left(\left|\mu_{20}\right|-\left|\mu_{02}\right|\right)}
\end{aligned}
$$

The two solutions are respectively the directions of main shaft ( $a$ axis) and $b$ axis:

$$
\alpha_{1}=1 / 2 \tan ^{-1}\left(\frac{2\left|\mu_{11}\right|}{\left|\mu_{20}\right|-\left|\mu_{02}\right|}\right), \alpha_{2}=1 / 2 \tan ^{-1}\left(\frac{2\left|\mu_{11}\right|}{\left|\mu_{20}\right|-\left|\mu_{02}\right|}\right)+\pi / 2 \text {. }
$$

For Ep. 14, calculate the second derivative of $\alpha$ :

$$
I_{L}^{\prime \prime}=2\left(\left|\mu_{20}\right|-\left|\mu_{02}\right|\right) \cos 2 \alpha+4\left|\mu_{11}\right| \sin 2 \alpha \text {. }
$$

Substitute the two solutions $\alpha_{1}, \alpha_{2}$ into Ep. 17, and if $I_{L}{ }^{\prime \prime}(\theta)>0, \theta \in\left(\alpha_{1}, \alpha_{2}\right), \theta$ will be the the main shaft direction. So the rotational angle can be calculated from Ep. 18:

$$
\varphi=\left|\alpha-\alpha^{\prime}\right| \text {. }
$$

Where, $\alpha, \alpha$ ' are respectively two axis direction angle of the main shaft in two images, as shown in Fig. 1. From what has been derived above, another important registration parameter needed in this paper is obtained.

After obtaining the rotation angle $\varphi$, the images are ready for registering, and the rotation transform for 2-D images is as the follow:

$$
\left[\begin{array}{lll}
x_{1} & y_{1} & 1
\end{array}\right]=\left[\begin{array}{lll}
x_{0} & y_{0} & 1
\end{array}\right]\left[\begin{array}{ccc}
\cos \varphi & -\sin \varphi & 0 \\
\sin \varphi & \cos \varphi & 0 \\
0 & 0 & 1
\end{array}\right] .
$$

Where, $\left(x_{0}, y_{0}\right)$ is the initial position of pixels, and $\left(x_{1}, y_{1}\right)$ is the position after transformation. As the name suggests, the point location of image pixel is defined as an integer, so after rotation transformation the pixel location need to round up. Inevitably, rounding up after rotation transformation, the image pixel can be repeated or missing, and this paper uses bilinear interpolation method [11] to fill up the vacancy. 
According to the distribution shape of color information and the quality relationships of two images, using the analogy deduction, the masses of two images decide the parameters of the transformation equation for the registration images.

According to Ep. 2 and what detailed above, the masses of the images are respectively $\left(x_{0}, y_{0}\right)$ and $\left(x_{1}, y_{1}\right)$ :

$$
\begin{aligned}
& x_{0}=\frac{\sum_{M} \sum_{N} x f(x, y)}{\sum_{M} \sum_{N} f(x, y)}=\frac{\left|m_{10}\right|}{\left|m_{00}\right|}, y_{0}=\frac{\sum_{M} \sum_{N} y f(x, y)}{\sum_{M} \sum_{N} f(x, y)}=\frac{\left|m_{01}\right|}{\left|m_{00}\right|} . \\
& x_{0}{ }^{\prime}=\frac{\sum_{M} \sum_{N} x^{\prime} f^{\prime}(x, y)}{\sum_{M} \sum_{N} f^{\prime}(x, y)}=\frac{\left|m_{10}\right|}{\left|m_{00}\right|}, y_{0}^{\prime}=\frac{\sum_{M} \sum_{N} y^{\prime} f^{\prime}(x, y)}{\sum_{M} \sum_{N} f^{\prime}(x, y)}=\frac{\left|m_{01}\right|}{\left|m_{00}\right|} .
\end{aligned}
$$

Among those, $m_{10}, m_{01}, m_{10}$ ', $m_{01}$ ', are the first-order matrixes and $m_{00}, m_{00}$ ' are the zero-order matrixes.

\section{Registration theory verification}

To test the reliability of the proposed methods above, this article will rotate a molten pool image a set of degrees, and the spinning process is shown as Fig. 3:

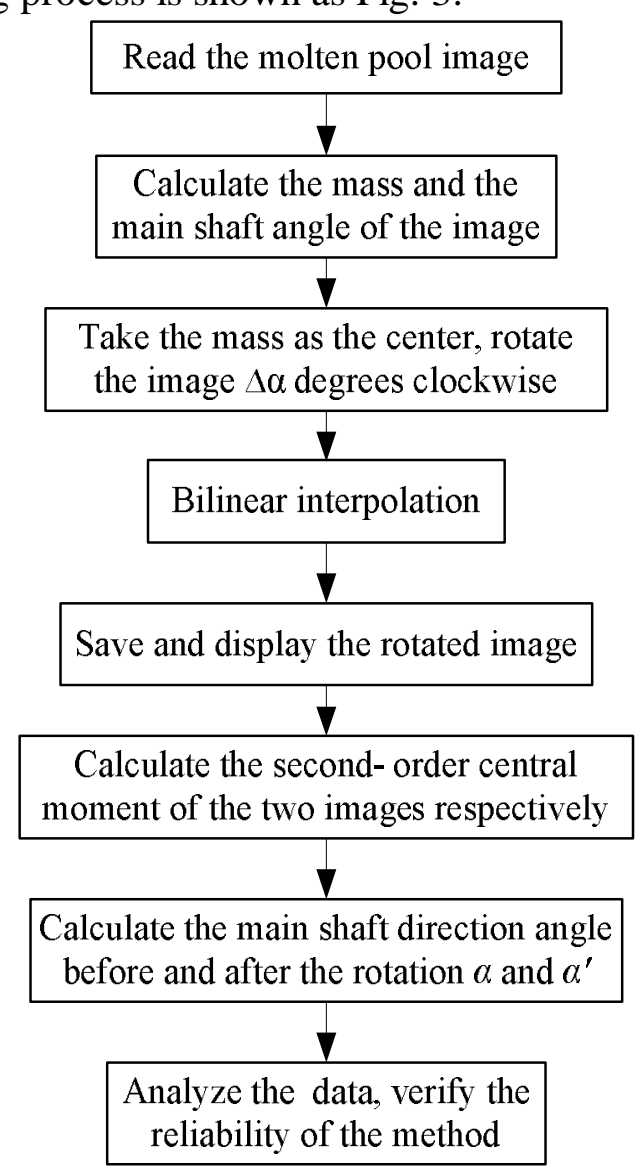

Fig. 3 Registration method reliability verification flow chart

According to the default result, if a molten pool image after overall clockwise or counterclockwise rotation of $\Phi$ degrees, according to that the ellipse-like main shaft direction of the molten pool is closely related to its pixels distribution, the main shaft direction will also rotate $\Phi$ degrees approximately. The following Table 1 and Table 2 show the relationship between the main shaft directions of molten pool images and the rotation degrees. 
Table 1 the main shaft directions and the rotation degrees (Fig. 1 clockwise)

\begin{tabular}{ccccccc}
\hline$\Phi /\left[^{\circ}\right]$ & 0 & 3 & 6 & 9 & 12 & 30 \\
\hline$\alpha /\left[^{\circ}\right]$ & 2.7814 & 5.8077 & 8.7922 & 11.8085 & 14.8276 & 32.8024 \\
$\Delta \alpha /\left[^{\circ}\right]$ & 0 & 3.0263 & 6.0108 & 9.0270 & 12.0462 & 30.0209 \\
\hline \multicolumn{7}{l}{ Table 2 the main shaft directions and the rotation degrees (Fig. 1 counterclockwise) } \\
\hline$\Phi /\left[^{\circ}\right]$ & 0 & -3 & -6 & -9 & -12 & -30 \\
\hline$\alpha /\left(^{\circ}\right)$ & 2.7814 & -0.1962 & -3.2438 & -6.2413 & -9.2638 & -27.1595 \\
$\Delta \alpha /\left[^{\circ}\right]$ & 0 & 2.9776 & 6.0252 & 9.0227 & 12.0452 & 29.9409 \\
\hline
\end{tabular}

According to the analysis above, there is a positive correlation between the main shaft directions of welding molten pool images and the rotation degrees. In the range of allowable error, $\Phi$ and the relative rotation degrees $\Delta \alpha$ calculated from $\alpha$ are almost the same.

\section{Registration experiment}

The corresponding flow chart of registration and registration results are shown below:

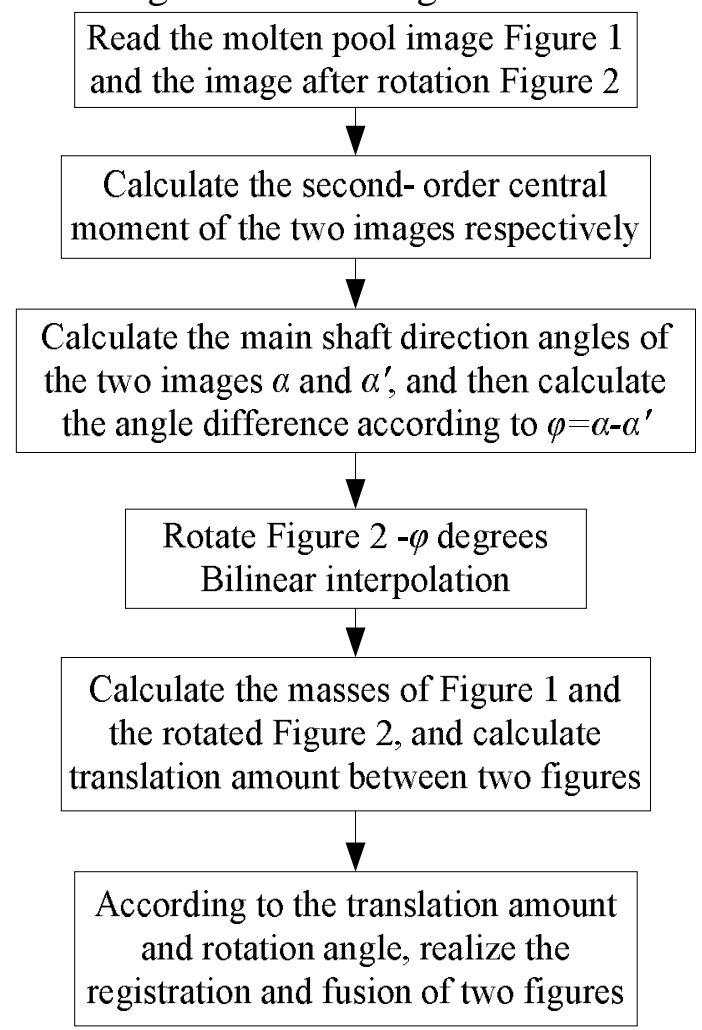

Fig. 4 The registration flow chart

High-speed image of the welding molten pool [9] is shown in Fig. 5. For the laser welding, stainless steel plates with $2 \mathrm{~mm}$ thickness have been welded with a $4 \mathrm{~kW}$ fast axial flow Q-mode $\mathrm{CO} 2$ laser. The recording images are made with an imaging system (type Phantom V4.1). The Fig. 6 is the molten pool image after $12^{\circ}$ rotation representing the other a molten pool image of the different perspective. 


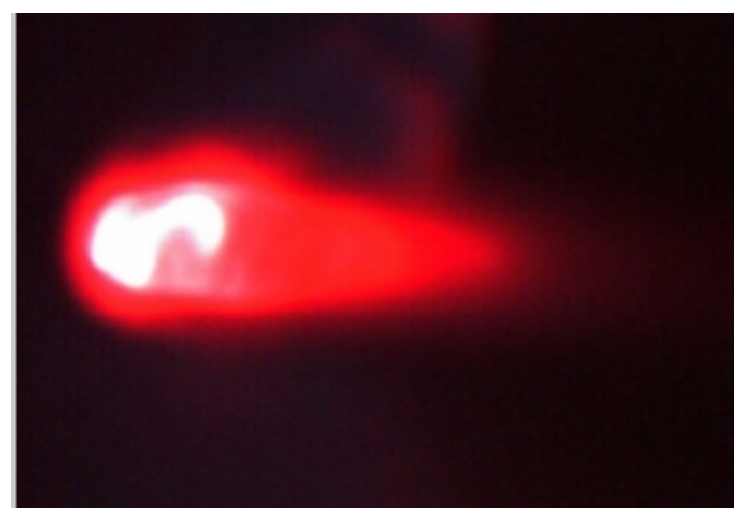

Fig.5 The molten pool image

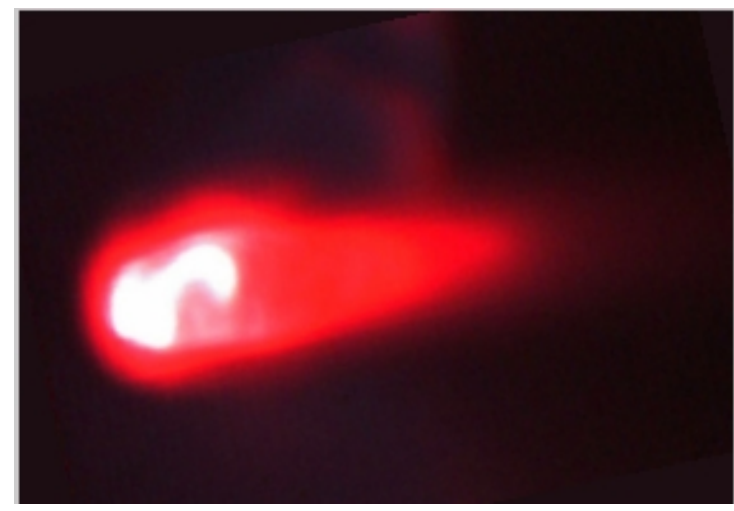

Fig.6 The molten pool image after $12^{\circ}$ rotation

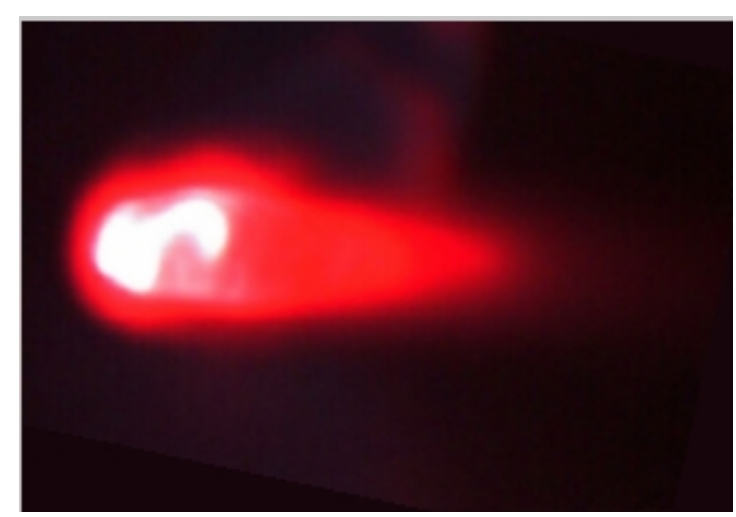

Fig.7 The reduction image after rotation algorithm and bilinear interpolation

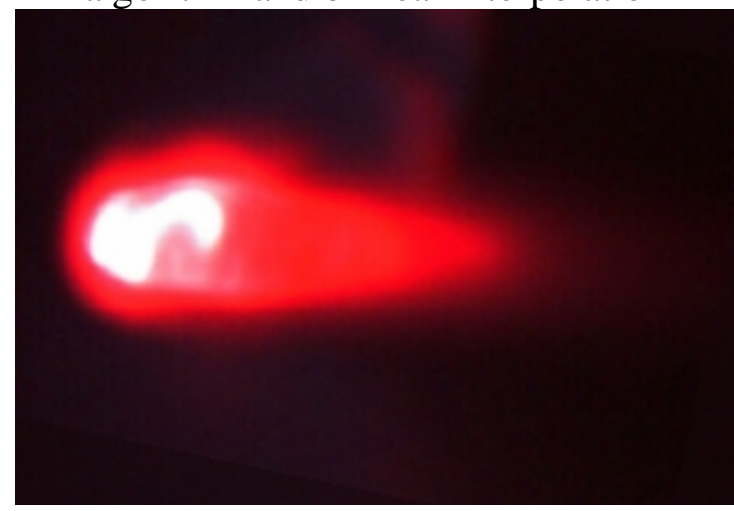

Fig.8 The registration and fusion results

As shown in these figures, after rotating $-\varphi$ degrees and bilinear interpolation (Fig. 7), the image is not distorted. And after registration and fusion (Fig. 8), the image becomes much clearer, and image information has become more abundant.

\section{Conclusions}

For the rich color information of laser welding images, this paper puts forward a new image registration method based on quaternion and geometric moment. This method is simple and effective. Compared with some registration methods that divided the $R, G, B$ the three channels into three single channels, this method does not produce color distortion and affect the outcome. Generally multi-mode images come from the same scene, and they have similar color distribution, so the method has certain feasibility for laser welding color image registration. It can provide accurate information for the following laser welding multispectral image segmentation and the pattern recognition and so on, and thus improve the accuracy and efficiency of the laser welding quality monitoring and identification.

\section{Acknowledgements}

This work was supported by the National Natural Science Foundation of China (61273024, 61305031), the Natural Science Foundation of Jiangsu Province (KB2012227), the "333 High-level Personnel Training Project" of Jiangsu Province, a Project Funded by the Priority Academic Program Development of Jiangsu Higher Education Institutions (PAPD), the "226 High-level Personnel Training Project" of Nantong city.

\section{References}

[1] Moradi M, Ghoreishi M, Frostevarg J, et al. An investigation on stability of laser hybrid arc welding[J]. Optics \& Lasers in Engineering, 2013, 51(4):481-487. 
[2] Xu G J, Wang H, Chang L L, et al. Characteristics of laser welding[J]. Electric Welding Machine, 2010, 40(11): 57-64.

[3] Wu B, Pang S Y, Duan A Q, et al. Numerical simulation of transient evolvement of molten pool in laser deep penetration welding[J]. Transactions of the China Welding Institution, 2010, 31 (10):1-4.

[4] Pang S Y, Chen L L, Yin Y J, et al. Simulations of transient keyhole and dynamic melt pool behaviors during laser welding[J]. Transactions of the China Welding Institution, 2010, 31(2):71-74.

[5] Zhang L J, Zhang J X, Gong S L. Recent advance in research for keyhole behavior during laser deep penetration welding process[J]. Laesr Journal, 2009, 30(5):3-5.

[6] Lu X, Ma H, Zhang B, et al. A review of algorithm research progress for non-rigid medical image registration[C]. International Conference on Consumer Electronics, Communications and Networks (CECNet), 2011. IEEE, 2011: 3863-3866.

[7] Li C, Chen Q, Qian W X. Registration algorithm of multispectral images based on cross cumulative residual entropy[J]. Infrared and Laser Engineering, 2013, 42(7): 1866-1870.

[8] Moghaddam M J, Soltanian-Zadeh H. Automatic Segmentation of Brain Structures Using Geometric Moment Invariants and Artificial Neural Networks[J]. Lecture Notes in Computer Science, 2009, 21:326-337.

[9] Wang H, Shi Y, Gong S, et al. Effect of pressure gradient driven convection in the molten pool during the deep penetration laser welding[J]. Journal of Materials Processing Technology, 2007, 184(1):386-392.

[10] Hua L. Medical Image Registration Based on Geometrical Algebra Theory [D]. Zhejiang University of Technology, 2013.

[11] Cai N, Zhang H Y, Zhang N, et al. Using bilinear interpolation and wavelet transformation to zoom images based on an error-amended sharp edge algorithm[J]. Laser \& Infrared, 2010, 40(5): 558-562. 